\title{
Postpartum Maternal and Neonatal Hospitalizations Among Women with HIV: A Population-Based Study
}

\author{
Erin M. Macdonald, Ryan Ng, Mark H. Yudin, ${ }^{2-4}$ Ahmed M. Bayoumi, ${ }^{1,3,5,6}$ Mona Loutfy, ${ }^{1,5-7}$ \\ Janet Raboud, ${ }^{1,8,9}$ Khatundi-Irene Masinde, ${ }^{7}$ Wangari E. Tharao, Jason Brophy," \\ Richard H. Glazier, ${ }^{1-3,5,9,12}$ and Tony Antoniou ${ }^{1,2,12}$
}

\begin{abstract}
Postpartum maternal and neonatal readmissions in the period shortly following birth are indicators of serious morbidity. We compared the risk of postpartum maternal and neonatal hospitalizations in women living with and without HIV in Ontario, Canada. We conducted a population-based study of pregnancies in Ontario between April 1, 2002 and March 31, 2011 using Ontario's administrative health care databases. Generalized estimating equations were used to derive adjusted odds ratios (aORs) and $95 \%$ confidence intervals (CI) for the association of HIV infection with postpartum maternal hospitalizations within 30 days of hospital discharge and neonatal hospitalizations within 30 and 60 days of hospital discharge. Between 2002/2003 and 2010/2011, 1,133,505 pregnancies were available for analysis, of which $634(0.06 \%)$ were to women living with HIV. The proportion of postpartum maternal hospitalizations $(2.8 \%$ versus $1.1 \%$; odds ratio 2.53 ; $95 \%$ CI 1.57 to 4.07) was higher among women with HIV. The multivariable adjusted odds ratio was $1.54(95 \% \mathrm{CI}$ 0.93 to 2.55 ). The proportions of neonates hospitalized within 30 (2.6\% versus $3.7 \%$; aOR $0.68,95 \%$ CI 0.42 to 1.10 ) and 60 days (4.9\% versus 4.9\%; aOR 0.86, 95\% CI 0.60 to 1.24 ) of discharge were similar among infants born to women with and without HIV. Women living with HIV are at a higher risk of postpartum maternal hospitalizations than women not living with HIV. The effect of HIV infection was attenuated by multivariable adjustment, suggesting that sociodemographic or health care factors are responsible for much of the difference in outcomes.
\end{abstract}

\section{Introduction}

$\mathbf{P}$ OSTPARTUM MATERnAL AND NEONATAL readmissions are costly and important indicators of maternal and infant morbidity. ${ }^{1-4}$ Existing research indicates that close to $4 \%$ of all infants are readmitted to hospital within 6 weeks of hospital discharge, while the risk of maternal readmission ranges from $0.98 \%$ to $2.16 \% .^{3,5-8}$ Factors associated with a heightened risk of neonatal readmission include preterm and small for gestational age births, low socioeconomic status, rural residence, and young maternal age. ${ }^{5}$ These findings are concerning in the context of HIV infection because women with HIV are more likely to live in low income neighborhoods and their children are at higher risk of adverse neonatal

\footnotetext{
${ }^{1}$ Institute for Clinical Evaluative Sciences, Toronto, Ontario, Canada.

${ }^{2} \mathrm{Li}$ Ka Shing Knowledge Institute, St. Michael's Hospital, Toronto, Ontario, Canada.

${ }^{3}$ Centre for Research on Inner City Health, St. Michael's Hospital, Toronto, Ontario, Canada.

${ }_{5}^{4}$ Department of Obstetrics and Gynecology, St. Michael's Hospital and University of Toronto, Toronto, Ontario, Canada.

${ }^{5}$ Institute of Health Policy, Management and Evaluation, University of Toronto, Toronto, Ontario, Canada.

${ }^{6}$ Department of Medicine, University of Toronto, Toronto, Ontario, Canada.

${ }^{7}$ Women's College Research Institute, Women's College Hospital, Toronto, Ontario Canada.

${ }^{8}$ Toronto General Research Institute, University Health Network, Toronto, Ontario, Canada.

${ }^{9}$ Dalla Lana School of Public Health, University of Toronto, Toronto, Ontario, Canada.

${ }^{10}$ Women's Health in Women's Hands Community Health Centre, Toronto, Ontario, Canada.

${ }^{11}$ Children's Hospital of Eastern Ontario and University of Ottawa, Ottawa, Ontario, Canada.

${ }^{12}$ Department of Family and Community Medicine, St. Michael's Hospital and University of Toronto, Toronto, Ontario, Canada.
}

(C) Erin M. Macdonald et al. 2105; Published by Mary Ann Liebert, Inc. This Open Access article is distributed under the terms of the Creative Commons Attribution Noncommercial License (http://creativecommons.org/licenses/by-nc/4.0/) which permits any noncommercial use, distribution, and reproduction in any medium, provided the original author(s) and the source are credited. 
outcomes than HIV-negative women. ${ }^{9}$ Similarly, the prevalence of risk factors for maternal rehospitalization, such as multiple gestation, cesarean delivery, and postoperative complications, is higher among women living with HIV relative to HIV-negative women. ${ }^{9-13}$

Despite the increased prevalence of risk factors for maternal and neonatal readmissions, no studies have examined the risk and causes of rehospitalization among women with HIV and their infants in the period immediately following discharge. Such data are important for ascertaining for which women postpartum surveillance is required and whether interventions targeting potentially preventable causes of readmission are required. Accordingly, we compared the risk of maternal and neonatal hospitalizations in the first 1 to 2 months following discharge among women living with and without HIV infection in Ontario.

\section{Materials and Methods}

\section{Setting}

We conducted a population-based study of pregnancies to women living with and without HIV in Ontario between April 1, 2002 and March 31, 2011. Ontario has a universal singlepayer, government administered health care system. This project was approved by the Research Ethics Board of Sunnybrook Health Sciences Centre, Toronto, Ontario.

\section{Data sources}

We used Ontario's administrative health databases, which were held securely in linkable files without any direct personal identifiers, and analyzed at the Institute for Clinical Evaluative Sciences (ICES). We identified all live births to all Ontario women between the ages of 18 and 49 years using the MOMBABY database, which deterministically links the Canadian Institute for Health Information Discharge Abstract Database inpatient admission records of all mothers and their newborn infants. Within this cohort, we identified women living with HIV using the Ontario HIV Database, an administrative data registry of Ontario residents with diagnosed HIV infection that was generated using a validated case-finding algorithm. ${ }^{14}$ The definition of three physician claims with an International Classification of Diseases, Ninth Revision code for HIV infection $(042,043,044)$ within a 3-year period has a sensitivity and specificity of $96.2 \%$ [95\% confidence intervals (CI) $95.2 \%$ to $97.9 \%$ ] and $99.6 \%$ (95\% CI $99.1 \%$ to $99.8 \%$ ), respectively, for identifying persons living with HIV.

We obtained hospitalization data from the Canadian Institute for Health Information Discharge Abstract Database, which contains detailed clinical information regarding all hospital admissions in Ontario. We used the Ontario Health Insurance Plan database to identify claims for physician services such as prenatal care, and used validated disease registries to define the presence of diabetes and hypertension. ${ }^{15,16}$ We obtained basic demographic data from the Registered Persons Database, a registry of all Ontario residents eligible for health insurance. We adjusted for differences in comorbidity by calculating the number of John Hopkins Aggregated Diagnosis Groups (ADG) for each woman, using the John Hopkins Adjusted Clinical Group system. ${ }^{17}$ Ecologic measures of neighborhood instability and deprivation, two dimensions of the Ontario Marginalization Index, were derived using the 2006 Canadian
Census. ${ }^{18}$ We determined the adequacy of prenatal care using the Revised-Graduated Prenatal Care Utilization Index (R-GINDEX). ${ }^{19}$ The R-GINDEX is a summary measure of prenatal care, and is calculated on the basis of the number of visits for prenatal care and the trimester care began, taking gestational age into account. Finally, we ascertained immigration status and world region of origin using the Citizenship and Immigration Canada Database. These databases were linked in a coded fashion using encrypted health card numbers, and are routinely used for population-based research examining maternal and neonatal outcomes.

\section{Outcomes}

The primary outcomes were postpartum maternal and neonatal hospitalizations. We defined postpartum maternal hospitalizations as women who were discharged alive and subsequently readmitted to hospital at least once within 30 days of discharge. Similarly, we defined neonatal rehospitalizations as infants discharged alive who were subsequently readmitted to hospital at least once within 30 days of discharge; in sensitivity analyses, we also analyzed readmissions within 60 days. Transfers between hospitals were not considered readmissions.

\section{Statistical analysis}

We compared baseline characteristics of mothers living with and without HIV using one-way analysis of variance for continuous variables, Cochrane-Armitage tests for ordinal variables, and chi-square tests for categorical variables. We used generalized estimating equations with a logit link function and an exchangeable correlation structure to derive adjusted odds ratios (aORs) and 95\% confidence intervals (CI) for the association of HIV infection with postpartum maternal and neonatal hospitalizations. To assess the contribution of demographic and clinical risk factors for rehospitalization, we adjusted for these factors in a hierarchical stepwise manner. Specifically, we adjusted first for maternal age and comorbidity (model 1), next added socioeconomic status (model 2), followed by immigration status (model 3), and finally adding adequacy of prenatal care, mode of delivery, multiple births, and induction of labor (model 4). This selection was guided by our a priori conceptualization of the relative importance of these risk factors and their likelihood of being correlated. We also adjusted for infant birth weight and preterm birth as continuous and categorical variables, respectively, in all neonatal hospitalization models. All analyses were conducted using SAS version 9.3 (SAS institute, Cary, NC).

\section{Results}

We identified 1,133,505 pregnancies during the 10-year study period, of which $634(0.06 \%)$ were among women living with HIV. Relative to women not living with HIV, women living with HIV were more likely to undergo caesarean section (38.5\% versus $27.8 \% ; p<0.001)$, deliver preterm $(15.8 \%$ versus $7.2 \% ; p<0.001$ ), and live in the most deprived neighborhoods (Table 1). With few exceptions, no differences were observed between women with and without HIV with respect to complications related to labor and delivery, although the median length of admission was about a day longer for both women with HIV and their infants (Table 1). 


\begin{tabular}{|c|c|c|c|}
\hline Characteristic & $\begin{array}{c}H I V \text { pregnancy } \\
\mathrm{n}=634\end{array}$ & $\begin{array}{c}\text { Non-HIV pregnancy } \\
\mathrm{n}=1,132,871\end{array}$ & $\mathrm{p}$-value \\
\hline Mean (SD) age at delivery (years) & $30.8(5.2)$ & $30.1(5.2)$ & 0.002 \\
\hline 18 to 34 years & $458(74.5)$ & $881,796(79.2)$ & 0.002 \\
\hline 35 to 49 years & $157(25.5)$ & $231,463(20.8)$ & \\
\hline \multicolumn{4}{|l|}{ Neighborhood income quintile } \\
\hline 1 (lowest) & $304(47.9)$ & $248,914(22.0)$ & \multirow{6}{*}{$<0.001$} \\
\hline 2 & $142(22.4)$ & $225,367(19.9)$ & \\
\hline 3 & $66(10.4)$ & $230,425(20.3)$ & \\
\hline 4 & 73 (11.5) & $234,477(20.7)$ & \\
\hline 5 & $45(7.1)$ & $189,324(16.7)$ & \\
\hline Missing & $\leq 5$ & $4,364(0.4)$ & \\
\hline \multicolumn{4}{|l|}{ Time since immigration } \\
\hline 0 to 4 years & $158(24.9)$ & $140,121(12.4)$ & \multirow[t]{4}{*}{$<0.001$} \\
\hline 5 to 9 years & $57(9.0)$ & $75,641(6.7)$ & \\
\hline 10 or more years & $90(14.2)$ & $76,500(6.8)$ & \\
\hline Long-term residents & $329(51.9)$ & 840,609 (74.2) & \\
\hline \multicolumn{4}{|l|}{ Region of birth } \\
\hline Africa or Caribbean & $254(40.1)$ & $39,226(3.5)$ & \multirow[t]{3}{*}{$<0.001$} \\
\hline Canada & $329(51.9)$ & 840,609 (74.2) & \\
\hline Other Regions & $51(8.0)$ & $253,036(22.3)$ & \\
\hline Median (IQR) maternal LOS (days) & $3.0(2.0$ to 4.0$)$ & $2.0(1.0$ to 3.0$)$ & $<0.001$ \\
\hline Median (IQR) infant LOS (days) & $3.0(2.0$ to 4.0$)$ & $2.0(1.0$ to 3.0$)$ & $<0.001$ \\
\hline Diabetes & $12(1.9)$ & 20,211 (1.8) & 0.836 \\
\hline Gestational diabetes & $37(5.8)$ & $57,007(5.0)$ & 0.355 \\
\hline Hypertension & $23(3.6)$ & 29,789 (2.6) & 0.116 \\
\hline Pregnancy-induced hypertension & $22(3.5)$ & $50,192(4.4)$ & 0.240 \\
\hline Multiple gestation & $19(3.0)$ & $19,849(1.8)$ & 0.017 \\
\hline Preterm birth & $100(15.8)$ & 81,047 (7.2) & $<0.001$ \\
\hline Median (IQR) gestational weeks at delivery & $38(37-40)$ & $39(38-40)$ & $<0.001$ \\
\hline Cesarean section & $244(38.5)$ & $314,723(27.8)$ & $<0.001$ \\
\hline Induced labor & $114(18.0)$ & $238,452(21.0)$ & 0.058 \\
\hline Median (IQR) prior parities & $0(0-1)$ & $0(0-1)$ & 0.084 \\
\hline Median (IQR) aggregated Diagnosis groups & $6(5-9)$ & $4(3-6)$ & $<0.001$ \\
\hline \multicolumn{4}{|c|}{ Revised graduated prenatal care Utilization index category } \\
\hline Adequate & $175(27.6)$ & $428,867(37.9)$ & \multirow[t]{5}{*}{0.001} \\
\hline Inadequate & $101(15.9)$ & $156,982(13.9)$ & \\
\hline Intensive & $54(8.5)$ & $61,597(5.4)$ & \\
\hline Intermediate & $304(47.9)$ & $484,055(42.7)$ & \\
\hline Missing & $0(0.0)$ & $1,370(0.1)$ & \\
\hline \multicolumn{4}{|l|}{ Material deprivation quintile } \\
\hline 1 (least deprived) & $68(10.7)$ & $296,868(26.2)$ & \multirow[t]{6}{*}{$<0.001$} \\
\hline & $72(11.4)$ & $233,052(20.6)$ & \\
\hline 3 & $98(15.5)$ & $213,667(18.9)$ & \\
\hline 4 & $117(18.5)$ & $190,959(16.9)$ & \\
\hline 5 & $261(41.2)$ & $183,836(16.2)$ & \\
\hline Missing & $18(2.8)$ & $14,489(1.3)$ & \\
\hline \multicolumn{4}{|l|}{ Residential instability quintile } \\
\hline 1 (least instability) & $77(12.1)$ & $303,577(26.8)$ & \multirow{6}{*}{$<0.001$} \\
\hline 2 & 72 (11.4) & $228,844(20.2)$ & \\
\hline 3 & $69(10.9)$ & $168,458(14.9)$ & \\
\hline 4 & $145(22.9)$ & $214,871(19.0)$ & \\
\hline 5 & $253(39.9)$ & $202,632(17.9)$ & \\
\hline Missing & $18(2.8)$ & $14,489(1.3)$ & \\
\hline \multicolumn{4}{|l|}{ Complications of labor and delivery } \\
\hline Premature rupture of membranes & $57(8.7)$ & $110,296(9.6)$ & 0.47 \\
\hline Obstructed labor: malposition & $16(2.5)$ & $44,755(3.9)$ & 0.06 \\
\hline Obstructed labor: other & $20(3.1)$ & $46,702(4.1)$ & 0.20 \\
\hline Umbilical cord complications & $59(9.0)$ & $144,945(12.6)$ & 0.007 \\
\hline Perineal laceration during delivery ${ }^{a}$ & $186(47.7)$ & $467,744(57.2)$ & $<0.001$ \\
\hline Maternal placental syndromes & $37(5.8)$ & $63,440(5.6)$ & 0.78 \\
\hline Fetal acid/base imbalance & $111(17.0)$ & 217,147 (18.8) & 0.24 \\
\hline Postpartum hemorrhage & $29(4.4)$ & $42,524(3.7)$ & 0.31 \\
\hline
\end{tabular}

${ }^{a}$ Denominator is vaginal deliveries only.

HIV, human immunodeficiency virus; SD, standard deviation; IQR, interquartile range; LOS, length of stay. 
Table 2. Association Between HiV and Postpartum and Postnatal Hospitalizations

\begin{tabular}{lccc}
\hline Outcome & Postpartum maternal hospitalization & 30-day neonatal hospitalization & 60-day neonatal hospitalization \\
\hline Model $1^{\mathrm{a}}$ & $1.92(1.18$ to 3.13$)$ & $0.64(0.40$ to 1.03$)$ & $0.85(0.59$ to 1.23$)$ \\
${\text { Model } 2^{\mathrm{b}}}_{\text {Model } 3^{\mathrm{c}}}$ & $1.83(1.13$ to 2.98$)$ & $0.63(0.39$ to 1.02$)$ & $0.84(0.58$ to 1.21$)$ \\
Model 4 $^{\mathrm{d}}$ & $1.67(1.03$ to 2.71$)$ & $0.70(0.44$ to 1.13$)$ & $0.91(0.63$ to 1.31$)$ \\
\hline
\end{tabular}

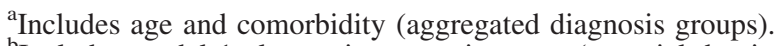

${ }^{\mathrm{b}}$ Includes model 1 plus socioeconomic status (material deprivation and residential instability).

${ }^{\mathrm{c}}$ Includes model 2 plus immigration status.

${ }^{\mathrm{d}}$ Includes model 3 plus adequacy of prenatal care, length of hospital stay, mode of delivery, multiple births, induction of labor, preterm birth (infant models only), and infant birth weight (infant models only).

The proportion of women who were hospitalized postpartum $(2.8 \%$ versus $1.1 \%$; odds ratio 2.53 ; $95 \%$ CI 1.57 to 4.07) was higher among women living with HIV relative to women without HIV. Overall, $44 \%$ of readmissions among women with HIV were related to infection of an obstetrical surgical wound or puerperal sepsis, compared to $10.7 \%$ of readmissions among women without HIV. Following adjustment for age and comorbidity, the risk of readmission within 30 days of discharge for women with HIV was attenuated (aOR 1.92; 95\% CI 1.18 to 3.13 ); this risk was further attenuated following adjustment for sociodemographic and health care variables (Table 2). In the fully adjusted model, the risk was no longer statistically significant (aOR 1,54; 95\% CI 0.93 to 2.55). Caesarean delivery was associated with postpartum maternal readmission (aOR 1.84; $95 \%$ CI 1.77 to 1.91 ). In a subgroup analysis, the risk of readmission was higher among women with HIV undergoing cesarean delivery (aOR 2.64; $95 \%$ CI 0.97 to 7.18 ) compared to HIV-negative women delivering by caesarean section (aOR $1.84 ; 95 \%$ CI 1.77 to 1.91 ).

The risk of neonatal hospitalizations among infants born to women with and without HIV was similar at both 30 days (2.6\% versus $3.7 \%$; aOR $0.68,95 \%$ CI 0.42 to 1.10 ) and 60 days $(4.9 \%$ versus $4.9 \%$; aOR 0.86 , $95 \%$ CI 0.60 to 1.24 ) following discharge (Table 2). Multivariable adjustment had little effect on the estimate of the adjusted odds ratio. Overall, 20 of the $42(47.6 \%)$ hospitalizations occurring within 60 days of discharge among infants born to women with HIV were attributable to infections, including pneumonia, sepsis, and urinary tract infections.

\section{Discussion}

We found that women with HIV were at higher risk of being readmitted within 30 days of discharge following childbirth relative to HIV-negative women. This risk was attenuated following multivariable adjustment, particularly when differences in health care characteristics such as caesarean delivery were considered. In contrast, we found no difference in the risk of infant postnatal hospitalizations within 30 and 60 days of discharge. Infection was the most common cause of readmission among women with HIV and their infants in the immediate postpartum period. Our study provides the first estimate of the risk of postpartum maternal and neonatal readmissions in women living with HIV.

We speculate that the increased proportion of maternal readmissions among women with HIV reflects the greater frequency of caesarean deliveries among these women. This reasoning is supported by earlier research documenting an
$80 \%$ increase in postpartum maternal rehospitalization risk among women with cesarean delivery ${ }^{3}$ and our finding that a large proportion of readmissions among women with HIV was attributable to obstetrical surgical wound infections. In addition, a meta-analysis of 10 studies found a $75 \%$ increased risk of wound infection among women with HIV undergoing a cesarean delivery relative to HIV-negative women. ${ }^{20} \mathrm{Im}$ munologic status at the time of cesarean delivery has been associated with subsequent complications in several, but not all studies. ${ }^{10,21-25}$ In light of these data, our findings suggest that preemptive measures may be required to reduce the burden of cesarean-related morbidity among women with HIV. Because vaginal delivery is an option for women with HIV with viral loads of 1,000 copies/ml or less, ${ }^{26}$ optimizing the antiretroviral management of pregnant women with HIV could decrease the need for cesarean deliveries and prevent morbidity related to this mode of delivery. For women requiring cesarean delivery, appropriate measures, such as antibiotic prophylaxis, limiting the number of vaginal examinations during labor, and postdischarge wound care in the community, may mitigate the risk of wound complications. ${ }^{3}$

The findings of this study are strengthened by the population-based nature of the data, thereby allowing us to include all women who delivered a baby in an Ontario hospital over the 9year study period. In addition, unlike previous studies, we were able to derive risk estimates that were adjusted for demographic and clinical characteristics that could influence the risk of postpartum readmission. However, some limitations of our study merit emphasis. As with all observational studies, it is possible that our findings partially reflect unmeasured confounders (e.g., breastfeeding rates, body mass index) or intergroup differences in the baseline risk of postpartum hospitalizations. In addition, unmeasured variables such as extent of immunosuppression and access to and adherence to antiretroviral therapy could account for within-group differences in the risk of infection among women with HIV.

We could not ascertain births that occurred outside the hospital or among women without provincial health insurance, which account for approximately $1.1 \%$ of all births in Ontario. ${ }^{27}$ We also could not ascertain births among women who were refugee claimants or who did not have provincial health insurance. As noted above, our databases do not include clinical information or reliable estimates of antiretroviral drug use. Accordingly, we supplemented our data with information drawn from other sources. Specifically, a recent study of persons with HIV in Ontario who receive care at specialized HIV clinics in the province found that $76.5 \%$ of women who were in care between the years 2001 and 2011 received combination antiretroviral therapy, of whom $83 \%$ 
had a suppressed viral load. ${ }^{28}$ Furthermore, mean CD4 cell counts at entry to HIV care between 2007 and 2010 were 406 cells $/ \mathrm{mm}^{3}$ among women aged 15 to 34 years and 340 cells $/ \mathrm{mm}^{3}$ among women aged 35 to 44 years. ${ }^{29}$ Finally, and of most relevance to the current study, we obtained supplemental data regarding antiretroviral therapy, viral load, and perinatal transmission from the Canadian Perinatal HIV Surveillance Program (extracted for 614 births to women living with HIV in Ontario for the period covered by our study), which indicates that $86.5 \%$ of women with HIV received combination antiretroviral therapy, and only $8.5 \%$ received no therapy during their pregnancy (Canadian Perinatal HIV Surveillance Program, personal communication). The proportion of women receiving antiretroviral therapy during pregnancy increased over time, from $83 \%$ in 2006 to over $90 \%$ for the period encompassing the years 2007 to $2009 .{ }^{29}$ Of the women on combination antiretroviral therapy, the risk of vertical transmission was $1.1 \%$. Viral load data were available for $91 \%$ of women during the period encompassing 2006 to 2011, of whom $82.8 \%$ attained virologic suppression below the limits of detection $(50$ copies $/ \mathrm{ml})$, with a further $10.3 \%$ being suppressed to less than 1,000 copies/ml (Canadian Perinatal HIV Surveillance Program, personal communication). Although we were not able to capture $\mathrm{CD} 4^{+}$count at delivery, these data indicate that most women were on antiretroviral therapy, virologically suppressed, and appropriately managed in terms of their HIV infection.

In conclusion, greater proportions of women with HIV are readmitted to hospital within 30 days of discharge following childbirth relative to HIV-negative women and infection of surgical obstetric wounds was the most common reason for these admissions. There was no significant difference in the proportion of infants born to women with and without HIV who were rehospitalized within 30 and 60 days of discharge. Further research aimed at optimizing postpartum infection control and characterizing the clinical characteristics of women with HIV readmitted to hospital will further clarify where areas for intervention exist for preventing such readmissions.

\section{Acknowledgments}

We would like to thank the investigators of the Canadian Perinatal HIV Surveillance Program for providing data regarding antiretroviral therapy and risk of vertical transmission for mother-infant pairs in Ontario.

All authors contributed to the concept and design of the study. Tony Antoniou, Erin Macdonald, and Ryan Ng acquired the data, and all authors were involved in the analysis and interpretation of the data. Tony Antoniou and Erin Macdonald drafted the article, and all authors were involved in critical revision of the article. All authors approved the article submitted for publication. Tony Antoniou, Erin Macdonald, and Ryan Ng provided administrative, technical, or material support. Tony Antoniou is the guarantor for the article.

This project was supported by a research operating grant from the Ontario HIV Treatment Network (Grant ROG G768) and by the Institute for Clinical Evaluative Sciences (ICES), which is funded by an annual grant from the Ontario Ministry of Health and Long-Term Care (MOHLTC). The sponsors had no role in the design or conduct of the study; in the collection, analysis, or interpretation of the data; or in the preparation, review, or approval of the article. The opinions, results, and conclusions reported in this article are those of the authors and are independent from the funding source. No endorsement by ICES or the Ontario MOHLTC is intended or should be inferred.

Tony Antoniou is supported by a New Investigator Award from the Canadian Institutes for Health Research-Ontario HIV Treatment Network. Mona Loutfy is the recipient of salary support from Women's College Hospital, the University of Toronto, and the Women's College Research Institute. Ahmed M. Bayoumi was supported by a Canadian Institutes for Health Research/Ontario Ministry of Health and Long-Term Care Applied Chair in Health Services and Policy Research. Janet Raboud is supported by an Ontario HIV Treatment Network Chair in Biostatistics and the Skate the Dream Fund, Toronto and Western Hospital Foundation. Richard H. Glazier is a Clinician Scientist in the Department of Family and Community Medicine of St. Michael's Hospital and the University of Toronto.

\section{Author Disclosure Statement}

No competing financial interests exist.

\section{References}

1. Roberts J, Sword W, Watt S, et al.: Costs of postpartum care: Examining associations from the Ontario mother and infant survey. Can J Nurs Res 2001;33:19-34.

2. Malkin JD, Broder MS, and Keeler E: Do longer postpartum stays reduce newborn readmissions? Analysis using instrumental variables. Health Serv Res 2000;35(5 Pt 2): 1071-1079.

3. Lydon-Rochelle M, Holt VL, Martin DP, and Easterling TR: Association between method of delivery and maternal rehospitalization. JAMA 2000;283:2411-2416.

4. Glazener CM, Abdalla M, Stroud P, et al.: Postnatal maternal morbidity: Extent, causes, prevention and treatment. Br J Obstet Gynaecol 1995;102:282-287.

5. Martens PJ, Derksen S, and Gupta S: Predictors of hospital readmission of Manitoba newborns within six weeks postbirth discharge: A population-based study. Pediatrics 2004; 114:708-713.

6. Bashiri A, Smolin A, Sheiner E, et al.: Maternal rehospitalization after singleton term vaginal delivery. J Matern Fetal Neonatal Med 2003;14:344-348.

7. Liu S, Heaman M, Joseph KS, et al.: Risk of maternal postpartum readmission associated with mode of delivery. Obstet Gynecol 2005;105:836-842.

8. Webb D and Robbins J: Mode of delivery and risk or postpartum re-hospitalization. JAMA 2003;289:46-47.

9. Antoniou T, Zagorski B, Macdonald EM, et al.: Trends in live birth rates and adverse neonatal outcomes among HIVpositive women in Ontario, Canada, 2002-2009: A descriptive population-based study. Int J STD AIDS 2014; 25:960-966.

10. Grubert TA, Reindell D, Kästner R, et al.: Rates of postoperative complications among human immunodeficiency virus-infected women who have undergone obstetric and gynecologic surgical procedures. Clin Infect Dis 2002;34: 822-830.

11. Lapaire O, Irion O, Koch-Holch A, et al.: Increased periand post-elective cesarean section morbidity in women 
infected with human immunodeficiency virus-1: A casecontrolled multicenter study. Arch Gynecol Obstet 2006;274: 165-169.

12. Marcollet A, Goffinet F, Firtion G, et al.: Differences in postpartum morbidity in women who are infected with the human immunodeficiency virus after elective cesarean delivery, emergency cesarean delivery, or vaginal delivery. Am J Obstet Gynecol 2002;186:784-789.

13. Fiore S, Newell ML, Thorne C, and the European HIV in Obstetrics Group: Higher rates of post-partum complications in HIV-infected than in uninfected women irrespective of mode of delivery. AIDS 2004;18:933-938.

14. Antoniou T, Zagorski B, Loutfy MR, et al.: Validation of case-finding algorithms derived from administrative data for identifying adults living with human immunodeficiency virus infection. PLoS One 2011;6:e21748.

15. Hux JE, Ivis F, Flintoft V, and Bica A: Diabetes in Ontario: Determination of prevalence and incidence using a validated administrative data algorithm. Diabetes Care 2002; 25:512-516.

16. Tu K, Campbell NR, Chen ZL, et al.: Accuracy of administrative databases in identifying patients with hypertension. Open Med 2007;1:e18-26.

17. Johns Hopkins University: Johns Hopkins ACG Case-Mix Adjustment System. Available at www.ach.jhsph.org.

18. Matheson FI, Dunn JR, Smith KLW, et al.: Development of the Canadian Marginalization Index: A new tool for the study of inequality. Can J Public Health 2012;103(Suppl 2): S12-S16.

19. Alexander GR and Kotelchuck M: Quantifying the adequacy of prenatal care: A comparison of indices. Public Health Rep 1996;111:408-418.

20. Calvert $\mathrm{C}$ and Ronsmans $\mathrm{C}$ : HIV and the risk of direct obstetric complications: A systematic review and meta-analysis. PLoS One 2013;8:e774848.

21. Maiques V, Garcia-Tejedor A, Diago V, et al.: Perioperative cesarean delivery morbidity among HIV-infected women under highly active antiretroviral treatment: A case-control study. Eur J Obstet Gynecol Reprod Biol 2010;153(1): 27-31.
22. Vimercati A, Greco P, Loverro G, et al.: Maternal complications after caesarean section in HIV infected women. Eur J Obstet Gynecol Reprod Biol 2000;90:73-76.

23. Semprini AE, Castagna C, Ravizza M, et al.: The incidence of complications after caesarean section in 156 HIV-positive women. AIDS 1995;9:913-917.

24. Watts DH, Lambert JS, Stiehm R, et al.: Complications according to mode of delivery among human immunodeficiency virus-infected women with CD4 lymphocyte counts of $<500 / \mu \mathrm{L}$. Am J Obstet Gynecol 2000;183:100-107.

25. Maiques-Montesinos V, Cervera-Sanchez J, et al.: Postcesarean section morbidity in HIV-positive women. Acta Obstet Gynecol Scand 1999;78:789-792.

26. Panel on Treatment of HIV-Infected Pregnant Women and Prevention of Perinatal Transmission: Recommendations for Use of Antiretroviral Drugs in Pregnant HIV-1-Infected Women for Maternal Health and Interventions to Reduce Perinatal HIV Transmission in the United States. Available at http://aidsinfo.nih.gov/contentfiles/lvguidelines/PerinatalGL .pdf. Accessed December 5, 2014.

27. Canadian Institute for Health Information: Too early, too small: A profile of small babies across Canada. Ottawa, CIHI, 2009.

28. Burchell AN, Gardner S, Light L, et al.: Engagement in HIV care among persons enrolled in a clinical HIV cohort in Ontario, Canada, 2001-2011 J Acquir Immune Defic Syndr [Epub ahead of print]; DOI:10.1097/QAI.0000000000000690.

29. Bayoumi AM, Degani N, Remis RS, et al.: HIV infection. In: Project for an Ontario Women's Health Evidence-Based Report: Volume 2 (Bierman AS, ed.). St. Michael's Hospital and the Institute for Clinical Evaluative Sciences, Toronto, Ontario, Canada, 2011.

Address correspondence to: Tony Antoniou 410 Sherbourne Street Toronto, Ontario M4X $1 K 2$ Canada

E-mail: tantoniou@smh.ca 